\title{
A System-Based Approach to Monitoring the Performance of a Human Neuromusculoskeletal System
}

\author{
Marcus Mussleman ${ }^{1}$, Deanna H. Gates ${ }^{2}$, and Dragan Djurdjanovic ${ }^{3}$ \\ ${ }^{1}$ Lam Research Corporation, Fremont, CA, 94538, USA \\ marcus.musselman@lamresearch.com \\ ${ }^{2}$ University of Michigan, Ann Arbor, MI, 48105, USA \\ gatesd@umich.edu \\ ${ }^{3}$ University of Texas, Austin, TX, 78712, USA \\ dragand@me.utexas.edu
}

\begin{abstract}
This paper presents a system-based method for monitoring a human neuromusculoskeletal (NMS) system. It is based on autoregressive models with exogenous inputs, which link surface electromyographic signals and joint kinematic variables in order to detect changes in system dynamics, as well as to assess joint level and muscle level contributions to those changes. Instantaneous energy and mean frequency of time frequency distributions of electromyographic signals were used as model inputs, while angular velocities of the monitored joints served as outputs. Slow temporal changes in the behavior of the entire system or individual joint models were tracked by analyzing one-step ahead prediction errors of the corresponding models over time. Finally, analysis of the recursively updated models, which tracked the NMS dynamics over time, was used to characterize these changes at the joint and muscular levels. The methodology is demonstrated on data recorded from 12 human subjects completing a repetitive sawing motion until voluntary exhaustion. Statistically significant decreasing trends in the similarities of the NMS models to those observed in the rested state were observed in all subjects. In addition, decreased joint response to muscle activity, as well as changes in the coordination and motion planning have been detected with all subjects, indicating their fatigue.
\end{abstract}

\section{INTRODUCTION}

Continuous monitoring of human body systems is only in its infancy, but with the advent of wearable electronics and

\footnotetext{
Musselman et al. This is an open-access article distributed under the terms of the Creative Commons Attribution 3.0 United States License, which permits unrestricted use, distribution, and reproduction in any medium, provided the original author and source are credited.
}

pervasive communications and computing, it is bound to grow significantly in the near future. The majority of methods for monitoring the performance of human body systems utilize only the outputs from the underlying systems to assess its performance. For these symptomatic monitoring methods, an anomalous output from a system is synonymous with an anomalous system. An underlying assumption for this paradigm is that the system inputs are stationary, which unfortunately is not true for almost all biomedical systems and thus applicability of symptomatic monitoring for long-term, continuous monitoring of human body systems is dramatically limited.

Alternatively, one can make use of both the inputs outputs of a system to formulate and track dynamic relationships between them, leading to the so-called system-based monitoring paradigm. Characterization and tracking of changes in the relationship between system inputs and outputs rather than just changes in its outputs enables one to monitor that system for a wide spectrum of inputs, i.e. without the requirement for the inputs to be stationary. Furthermore, it allows one to determine if changes in the system behavior are indeed due to changes in the system dynamics, or due to unusual inputs for which a credible model of system dynamics is not established. Thus, system based monitoring approach carries significant benefits over the symptomatic one.

While system-based performance monitoring remains unfeasible for many biomedical systems, the neuromusculoskeletal (NMS) system is ripe for this paradigm shift because its inputs and outputs are more or less measurable and a significant amount of work has already been done to relate the two. Namely, joint torques arise from muscle contractions, which are induced via 
electrical signals from the nervous system. Effects of electrical stimulations of the muscles are indirectly measurable through electromyography (EMG) signals, which can therefore be seen as inputs into the NMS system. Furthermore, joint torques and motion variables (angular positions and velocities) constitute the outputs from the NMS system and are also measurable via dynamometers and motion capture systems. A system-based monitoring scheme for the NMS system could enable continuous tracking and characterization of changes in the NMS dynamics, without the need to necessarily prescribe motion patterns that a subject needs to perform. Such capability could facilitate personalizing and customizing of training regimens for athletes and patients undergoing rehabilitation by prescribing exercises that target the muscles and joints with the greatest deficits for a given person, at a given time, as assessed via the system model. Furthermore, therapeutic exercise regimens for patients with NMS impairments can thus be more precisely tailored toward returning the patient to nominally healthy NMS dynamics, and to more reliably indicate when to stop training or rehabilitation before the onset of injury. In other words, system-based monitoring of human NMS system performance could facilitate conditionbased health management practices with our NMS systems similar to how we today pursue condition-based health management of our machines.

Measurements of EMG signals and relevant kinematic and dynamic variables have been used extensively in literature to construct dynamic models of various portions of the NMS system (thorough reviews of the relevant literature can be found in the works by Lee, Glueck, Fiume and Jackson (2010) and Viceconti, Testi, Taddei, Martinelli, Clapworthy and Jan (2006). Generally, these models were derived using first principles or data-driven approaches to estimate the relationship between EMG and joint output variables for fingers, various parts of the arm, leg joints, as well as spine.

As illustrated in Figure 1, these models try to conect the EMG signals with the corresponding muscle forces, and further with joint torque and motion variables. The muscle force generation is tackled using various methods for processing and transforming EMG signals. Many EMG processing approaches seek to emulate the underlying physics of neural control and action potential propagation (Keynes, Aidley and Huang, 2010). These techniques are reviewed by Buchanan, Lloyd, Manal and Besier (2005) and include normalization based on the force-length and forcevelocity relations, as well as first order chemical kinetics equations. On the other hand, numerous purely data-driven EMG processing techniques were used for modeling of NMS dynamics, including linear envelope processing (Hashemi, Hashtrudi-Zaad, Morin and Mousavi, 2010), lowpass filtering (Koike and Kawato, 2010), extraction of mean or median power frequency from EMG spectra $(\mathrm{Xu}$, Zhang and Pang, 2010), or Shannon entropy from EMG spectrograms (Arslan, Adli, Akan and Baslo, 2010).
When it comes to the joint geometry and dynamics, most models in the literature handle them via the model structure itself. One approach is the path of physics and physiology based first principles, as seen in the paper by Buchanan et al., (2005). Data driven approaches are the alternative approach within which neural networks (Song and Tong, 2005) and regression-based techniques (Potvin and Brown, 2004) are the two overwhelmingly dominant structures utilized in the literature.

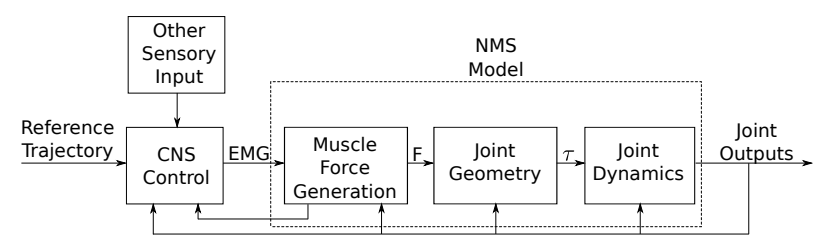

Figure 1. Block Diagram of constituent parts of NMS system (Keynes et al., 2010).

Despite the aforementioned advancements in NMS system modeling, most applications do not fully utilize the information about the temporal changes in the frequency content of EMG signals. On the other hand, temporal changes in neuronal excitation frequency are highly important for NMS function because the very nature of neural communication with muscles can be characterized by both amplitude and frequency modulation (Keynes et al., 2010). Furthermore, monitoring the NMS system performance has been approached from a purely symptomatic perspective, relying on tracking the behavior of either the EMG signatures (Dingwell, Napolitano and Chelidze, 2007), or joint kinematic trajectories (Segala, Gates, Dingwell and Chelidze, 2011), independently. A system-based approach to monitoring the NMS system based on dynamic models relating EMG signals with joint kinematic variables has not yet been posed.

This paper seeks to rectify the abovementioned shortcomings by using advanced signal processing to extract new features from EMG signals, which will then be used to build dynamic models that permit tracking and characterization of changes in the NMS system. The rest of the paper is organized as follows. Section 2 describes the signal processing and modeling techniques used to relate the EMG signals to joint kinematics. Section 3 details experimental data collected from human subjects and presents the results of applying the newly proposed systembased monitoring method on that data. Finally, Section 4 outlines the contributions of this paper and avenues for future work.

\section{Methods}

The newly proposed NMS monitoring method tracks changes in the dynamic relationships between inputs derived from EMGs and outputs obtained from joint kinematic measurements. The general framework includes: 
- Modeling joint kinematic variables as a function of EMG signatures;

- Tracking of statistical characteristics of modeling errors over time to capture degradation (changes) in the NMS system behavior;

- Monitoring of changes in the NMS model parameters over time, enabling assessments of changes in the effects of individual muscles on the NMS system dynamics;

\subsection{Modeling Considerations}

The daunting task of establishing a model between EMG measurements and joint kinematics necessitates overcoming of two pervasive challenges. Firstly, EMG is a noisy signal, which, at best, is a measure of the collective nerve and muscle depolarization underneath the electrodes. This difficulty has given rise to many attempts to use advanced signal processing techniques to extract useful and informative features from the EMG signals. The second difficulty is that even with perfect neural command information, the NMS system is inherently a complex, nonlinear system with multiple inputs and outputs (MIMO) (Keynes et al., 2010). Hence, our explorations of system based monitoring of the NMS system needed to cope with challenges of EMG noise and complexity, model structure and parameter estimation.

\subsubsection{EMG Processing and Feature Extraction}

Neuronal communications within the body are characterized by the frequency, amplitude, and phase of the neuron action potential train. More specifically, muscle force amplitude, as a function of motor neuron activation, is characterized by the so-called "twitch response" (Keynes et al., 2010). This results in an increase in mean muscle force with increasing neuronal activation frequency in isometric contraction. The twitch response also results in a nonlinear, low-pass filter response between the neuronal activation and muscle force generation (Itoh, Atayaki, Mita, Watanabe and Nonaka, 2013). Furthermore, isometric muscle activation, and hence muscle force production via the Hill-type model, has been shown to be a piecewise linear and logarithmic function of neuronal excitation (Manal, 2003). Given these reasons, it is pertinent for any model connecting surface EMG (sEMG) signals with joint kinematics to incorporate information on the temporal behavior of both the amplitude and the frequency of the sEMG signal.

Consequently, a joint time-frequency signal representation of sEMG signals was pursued. More specifically, we utilized Cohen's class of time-frequency distributions (TFD) to gain concurrent insight into how SEMG signal energy varies in both time and frequency (Cohen, 1995). The TFD, $C(t, \omega)$ of a signal, $s(t)$ is calculated as

$$
\begin{gathered}
C(t, \omega)=\frac{1}{4 \pi^{2}} . \\
\iiint_{-\infty}^{\infty} s^{*}\left(u-\frac{\tau}{2}\right) s\left(u+\frac{\tau}{2}\right) \phi(\theta, \tau) e^{-j(\theta(t-u)+\tau \omega)} d \tau d u d \theta
\end{gathered}
$$

where $s^{*}(t)$ denotes the complex conjugate of $s(t)$ and $\phi(\theta, \tau)$ is the TFD kernel. This signal processing approach was adopted because it does not suffer from time-domain and frequency domain resolution tradeoffs, as do the more traditional wavelets-based and spectrogram-based timefrequency signal representations. In addition, developments in late 1980s and 1990s led to time-frequency kernels that yield distribution with superior mathematical properties of strong time and frequency support, upholding of time and frequency marginals, as well as conditional expectations calculated based on those TFDs evaluating to the instantaneous frequency and group delay of the signal ${ }^{1}$ (Jeong and Williams, 1992; Cohen, 1995).

In this paper, we utilized the binomial kernel (Jeong and Williams, 1992), which is a signal independent member of the so-called reduced interference distribution family of kernels. The signal independent nature of this kernel enables a faster calculation of TFDs compared to signal dependent kernels, while delivering the aforementioned desirable mathematical properties, along with signal filtering based on the suppression of time-frequency cross-terms. Because of those favorable mathematical properties, the binomial kernel based TFD could subsequently be used to effectively extract time-dependent signatures that are indicative of the instantaneous amplitude and frequency of the sEMG signal. Specifically, once the binomial TFD $C(\omega \mid t)$ of a sEMG signal is calculated, for each moment in time $t$, the corresponding instantaneous energy, $\left\langle f^{0} \mid t\right\rangle$, and instantaneous frequency, $\left\langle f^{1} \mid t\right\rangle$, of the signal could be calculated as follows

$$
\begin{gathered}
\left\langle f^{0} \mid t\right\rangle=\int_{-\infty}^{\infty} C(\omega \mid t) d \omega \\
\left\langle f^{1} \mid t\right\rangle=\int_{-\infty}^{\infty} f C(\omega \mid t) d \omega
\end{gathered}
$$

where

$$
C(\omega \mid t)=\frac{C(t, \omega)}{\int_{0}^{\infty} C(t, \omega) d \omega}
$$

denotes the conditional distribution of signal's energy at time $t$. Inspecting Eqs. (1)-(3) reveals that the instantaneous energy and frequency of the signal are expressed as conditional expectations, obtained using the binomial TFD as a two-dimensional probability density function of the signal energy in time and frequency ${ }^{2}$.

\footnotetext{
${ }^{1}$ Please note that it is possible to show that well-known spectrograms are also a member of Cohen's class of TFDs, with its kernel being the so-called ambiguity function of the window used to produce that spectrogram (Cohen, 1995). Nevertheless, spectrograms only have some of the aforementioned desirable mathematical properties.

${ }^{2}$ Once again, this is possible because binomial kernel TFDs have the properties of strong time and frequency support, time and frequency
} 
These signatures were pursued because decreases in instantaneous frequencies (Allison and Fujiwara, 2002), and increases in instantaneous intensities of sEMG signals (Disselhorst-Klug, Schmitz-Rode and Rau, 2009), are considered as classic indicators of muscle fatigue in the literature. Consequently, time series of instantaneous energies and frequencies, extracted from each of the relevant sEMG signals were used as the inputs for the dynamic model predicting the joint kinematics. The model form and calculation of its parameters are discussed next.

\subsubsection{Vectorial ARX Modeling of the NMS System}

Several portions of the NMS system can be viewed as serial chain manipulators, as illustrated in Figure 2 (Asfour, Berns, Schilling and Dillman, 1999). Following Artemidis and Kyirakopoulos, (2009), representing a human limb as a serial chain manipulator permits the formation of equations of motion as

$$
\ddot{\vec{\theta}}=\mathbf{D}^{-1}(\vec{\theta})\left[-\mathbf{C}(\dot{\vec{\theta}}, \vec{\theta}) \dot{\vec{\theta}}-g(\vec{\theta})+\vec{\tau}_{\text {muscle }}\right]
$$

where:

- $\vec{\theta}$ is the vector of joint angles;

- $\mathbf{D}(\vec{\theta})$ is the inertia matrix;

- $\quad \mathbf{C}(\dot{\vec{\theta}}, \vec{\theta})$ describes centrifugal and Coriolis effects;

- $g(\vec{\theta})$ arises from the potential energy of the robot;

- $\vec{\tau}_{\text {muscle }}$ is the vector of torques exerted by the muscles;

In order to approach the system-based monitoring problem from an analytically tractable perspective, in this paper we assume a linear, discrete form to approximate Eq. (4). Furthermore, in order to approximate the effects of the twitch response, the joint torque, $\vec{\tau}_{\text {muscle }}$, caused by muscular contraction will be modeled as a linear dynamic transformation of EMG features, $\left\langle f^{0} \mid t\right\rangle$ and $\left\langle f^{1} \mid t\right\rangle$, yielding the model in the form of a vectorial autoregressive model with exogenous inputs (vARX)

$$
\begin{gathered}
\dot{\vec{\theta}}[k]=\boldsymbol{\alpha} \dot{\vec{\theta}}[k-1]+\boldsymbol{\beta} \vec{\theta}[k-1]+ \\
+\sum_{g=1}^{G}\left[\vec{\gamma}_{g}\left\langle f^{0} \mid\left[k-\tau_{g}^{E M}\right]\right\rangle_{g}+\vec{\delta}_{g}\left\langle f^{1} \mid\left[k-\tau_{g}^{E M}\right]\right\rangle_{g}\right]+\vec{\varepsilon}[k]
\end{gathered}
$$

where:

- $k$ denotes the sample index of the relevant signal, meaning that $\dot{\vec{\theta}}[k]=\dot{\vec{\theta}}(k T), \vec{\theta}[k]=\vec{\theta}(k T)$ and so on, where $T$ denotes the sampling interval.

- $\boldsymbol{\alpha}$ is a matrix of autoregressive coefficients relating joint velocities to one another;

- $\boldsymbol{\beta}$ is a matrix of exogenous coefficients describing the effect of joint angles on the joint velocities;

marginals, as well as relevant conditional expectations evaluating to the instantaneous frequency and group delay of the signal.
- $\vec{\gamma}=\left[\vec{\gamma}_{1}, \ldots, \vec{\gamma}_{G}\right]$ is the matrix of exogenous coefficients describing the effect of sEMG instantaneous energies on joint velocity;

- $\overrightarrow{\boldsymbol{\delta}}=\left[\vec{\delta}_{1}, \ldots, \vec{\delta}_{G}\right]$ is the matrix of exogenous coefficients describing the effect of sEMG instantaneous frequencies on joint velocity;

- $\tau^{E M}=\left[\vec{\tau}_{1}, \ldots, \vec{\tau}_{G}\right]$ is a vector of electromechanical delays (EMDs), describing the delays from neural and muscle activation picked up by EMG electrodes, to the start of muscle force generation for all the $G$ muscles. Existence of these delays is well documented in the literature (Corcos, Gotlieb, Latash, Almeida and Agarwal, 1992)

- $\left\langle f^{0} \mid t\right\rangle_{g}$ is the instantaneous energy for the $g^{\text {th }}$ muscle;

- $\left\langle f^{1} \mid t\right\rangle_{g}$ is the instantaneous frequency for the $g^{\text {th }}$ muscle;

- $\vec{\varepsilon}[k]$ is the vector of noise terms due to linearization errors and un-modeled effects;

The authors are aware that linearity of model (5) is a strong and restrictive assumption, but, as will be shown in Section 3, analytical tractability of (5) led to some interesting insights enabled by the ability to efficiently adapt the model to the changes in the NMS system that occurred as the subject exercises progressed.

\subsubsection{Estimation of Electromechanical Delay}

Muscles are constructed of varying proportions of fast and slow twitch muscles and the EMD is positively correlated to the proportion of slow twitch fibers in the muscle. Therefore, the value of the EMD must be determined for each muscle independently, which was accomplished in this paper using a genetic algorithm (GA).

The GA minimized the Akaike information criterion (AIC) for the MIMO model (5), as proposed by Hurvich and Tsai (1991). Each candidate solution of the GA was represented by a chromosome whose $i^{\text {th }}$ element corresponds to the $i^{\text {th }}$ muscle EMD, as illustrated in Figure 3. Following Corcos et al. (1992), the chromosome elements are constrained to represent EMD times between $10 \mathrm{~ms}$ and $100 \mathrm{~ms}$. For a given vector of EMDs, the remaining model parameters were obtained by linear least squares estimation, allowing one to assess the fitness of the corresponding model via its AIC.

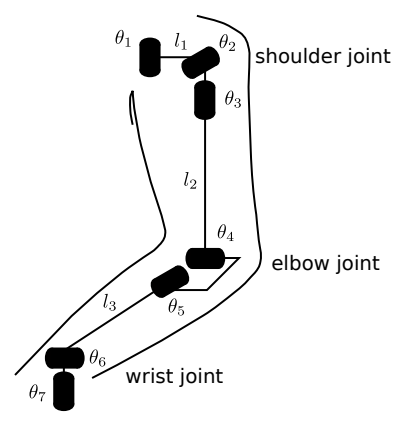

Figure 2. Example of the human arm expressed as a serial chain manipulator (Asfour et al., 1999). 


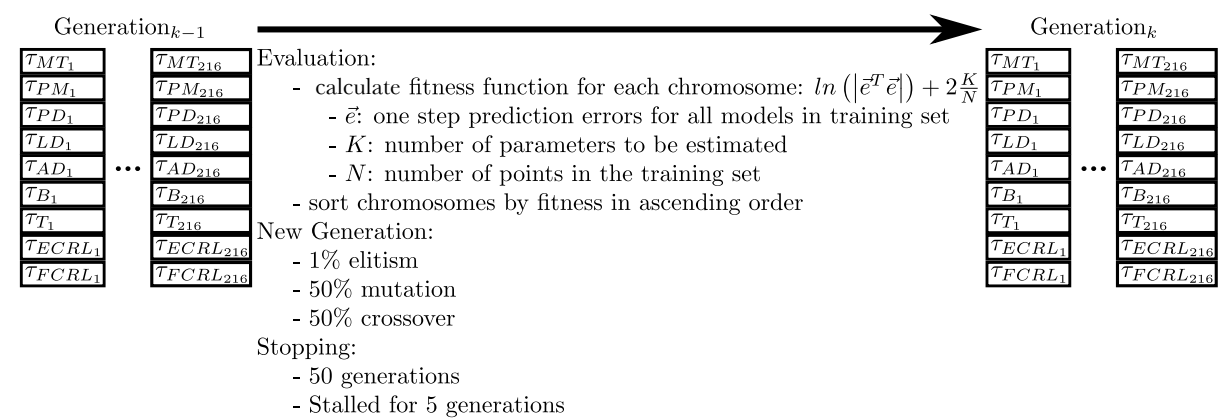

Figure 3. Illustration of the $(\boldsymbol{k}-\mathbf{1})^{\text {st }}$ (left) and the $\boldsymbol{k}^{\text {th }}$ generation (right) of the GA, where the $\boldsymbol{i}^{\text {th }}$ chromosome element represents the delay for the $\boldsymbol{i}^{\text {th }}$ muscle.

The GA population size was set to 216 with a $1 \%$ elite carryover from each generation to the next. The remaining $99 \%$ of each population was produced by crossover and mutation in even measures and implemented as proposed by Deep, Singh, Kansal and Mohan (2009). To inspire diverse solutions, the GA was run in 10 parallel sessions and a $20 \%$ migration of the best solutions from each session occurred every 5 generations. All GA parameters in this paper were selected ad hoc, after a few trial and error sessions, though one can find focused studies on systematic selection of GA parameters that maximize the resulting convergence speed and exploratory capabilities (De Jong, 1975; Bäck, 1995).

\subsection{Model Based Characterization of NMS Performance Degradation}

For each subject, a vARX model was trained on a data set believed to be representative of his/her least degraded state. This model will be referred to as the fresh model and this data set will be called the fresh data set. Once the fresh model is identified, the distribution of one-step ahead prediction errors it makes on the fresh data set is modeled using a Gaussian Mixture Model (GMM). The GMM form is used because of its universal approximation properties and the ability to efficiently update its parameters as new data become available (Lindsay, 1995). The distribution of errors made by the fresh model on the fresh data set will be referred to as the fresh error distribution.

As the newly measured data are presented to the model and new one-step prediction errors become available, the GMM of modeling errors is continuously updated via re-estimation of its parameters using the most recently observed errors. This yields the distribution, which we will refer to as the updated error distribution.

If the dynamic relationship between the inputs and outputs of the monitored system is unchanged, the updated distribution of modeling errors should be very similar to the fresh distribution. However, the updated distribution will move and change shape as the NMS system dynamics change due to e.g. fatigue or injury, causing the aforementioned overlap between distributions to be smaller than that observed during degradation-free operation. Matusita's overlap coefficient between the fresh distribution, $p_{1}(\vec{x})$, and the updated distribution, $p_{2}(\vec{x})$, of modeling residuals. defined as

$$
G F I=\int \sqrt{p_{1}(\vec{x}) p_{2}(\vec{x})} \mathrm{d} \vec{x}
$$

was used to measure and track the similarity between the two distributions over time (Matusita, 1955). This metric, referred to in the rest of the paper as the Global Freshness Index (GFI), varies between 0 and 1 , with values near 1 indicating high similarity between the most recent behavior dynamics and that observed on the fresh data set. Conversely, GFI values near 0 indicate little similarity between the most recent behavior dynamics and the behavior observed in the fresh data set.

Let us now note that both the fresh and updated error distributions are multivariate probability density distributions, with each dimension corresponding to one step prediction errors of an individual joint velocity. Therefore, the marginals of these multivariate distributions contain information about errors corresponding to the models of each individual joint velocity in Eq. (5). Consequently, Matusita's overlaps can be evaluated on the marginals of the fresh and updated error distributions, yielding individual Joint Freshness Indices (JFI), as illustrated in Figure 4. In this manner, JFIs can be used to assess relative contributions of each individual joint to the degraded behavior of the NMS system.

Next, let us gain further understanding of changes in the NMS system dynamics via characterization of changes in the influences of relevant muscles on the NMS dynamics. 


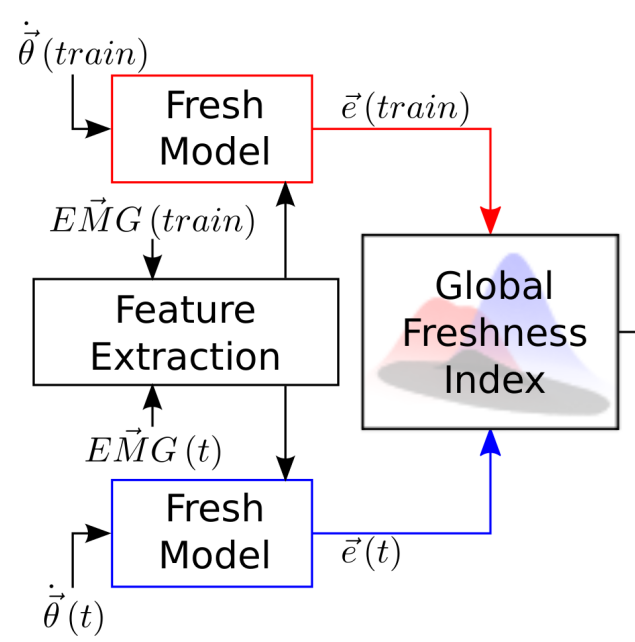

Joint 1

Freshness

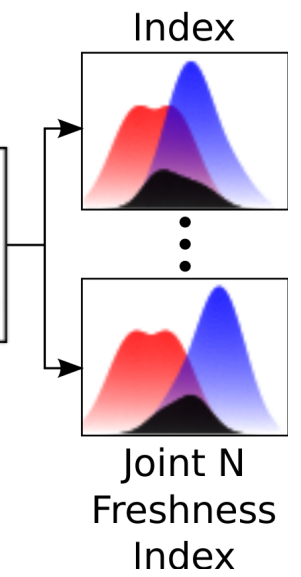

Figure 4. Flow chart of model based monitoring and joint-level characterization of NMS system degradation.

\subsection{Characterizing Changes in NMS Performance at the Joint Dynamics and Muscle Levels}

As new EMG features and joint velocities are observed, the linear vARX model (5) that relates them can be efficiently updated to track changes in the underlying dynamics. In this paper, the parameter matrices $\boldsymbol{\gamma}, \boldsymbol{\beta}$ and $\boldsymbol{\alpha}$ of the updated model are adapted using a recursive least squares algorithm with a forgetting factor, thus describing the dynamics of the most recent NMS system behavior ${ }^{3}$.

At any time moment, the similarity of dynamic responses from an input to an output between the fresh model and the updated model was assessed by comparing the frequency responses of the relevant transfer functions emanating from the MIMO model (5). Using forward Euler integration (Atkinson, 1989), Eq. (5) is cast into state space form

$$
\begin{aligned}
& {\left[\begin{array}{l}
\vec{\theta} \\
\overrightarrow{\dot{\theta}}
\end{array}\right][k+1]=\mathbf{A}\left[\begin{array}{l}
\vec{\theta} \\
\overrightarrow{\dot{\theta}}
\end{array}\right][k]+\mathbf{B}\left[\begin{array}{l}
\vec{u} \\
\vec{v}
\end{array}\right][k]+\left[\begin{array}{l}
\overrightarrow{0} \\
\vec{\varepsilon}
\end{array}\right][k]} \\
& \vec{y}[t]=\mathbf{C}\left[\begin{array}{l}
\vec{\theta} \\
\overrightarrow{\dot{\theta}}
\end{array}\right][k] \\
& \mathbf{A}=\left[\begin{array}{ll}
\mathbf{I} & T \mathbf{I} \\
\boldsymbol{\beta} & \boldsymbol{\alpha}
\end{array}\right] \\
& B=\left[\begin{array}{cc}
0 \\
{\left[\begin{array}{ll}
\gamma & \delta
\end{array}\right]}
\end{array}\right] \\
& \mathbf{C}=\left[\begin{array}{ll}
\mathbf{0} & \mathbf{I}
\end{array}\right]
\end{aligned}
$$

where:

- $\quad \mathbf{I}$ is the identity matrix

- $\mathbf{0}$ is a zero matrix

- $\vec{u}$ is the vector of muscle instantaneous energies, $\left\langle f^{0} \mid t\right\rangle$, delayed by their respective EMDs;

- $\vec{v}$ is the vector of muscle instantaneous frequencies, $\left\langle f^{1} \mid t\right\rangle$, delayed by their respective EMDs;

\footnotetext{
${ }^{3}$ Please note that EMDs were not adapted, which enabled such a tractable model adaptation.
}

- $\left[\begin{array}{l}\vec{\theta} \\ \overrightarrow{\dot{\theta}}\end{array}\right][k]=\left[\begin{array}{l}\vec{\theta}[k] \\ \overrightarrow{\dot{\theta}}[k]\end{array}\right]$ is the state vector at sample $k$

Following Franklin, Powel and Emami-Naeini (2006), the corresponding matrix of transfer functions, $\mathbf{H}(z)$, can now be calculated as

$$
\mathbf{H}(z)=\mathbf{C}(z \mathbf{I}-\mathbf{A})^{-1} \mathbf{B}
$$

For the element $\mathbf{H}_{i, k}^{(f)}(z)$ in the $i^{\text {th }}$ row and the $k^{\text {th }}$ column of the transfer matrix $\mathbf{H}^{(f)}(z)$ of the fresh model, $\mathbf{H}_{i, k}^{(f)}\left(e^{j \omega}\right)$ would correspond to the frequency response of the fresh model between input $k$ and output $i$, where $\omega \in\left[0 . \omega_{N}\right]$ and $\omega_{N}$ is the Nyquist frequency. Similarly, for the element $\mathbf{H}_{i, k}^{(u)}(z)$ in the $i^{t h}$ row and $k^{t h}$ column of the transfer matrix $\mathbf{H}^{(\boldsymbol{u})}(z)$ of the updated model, $\mathbf{H}_{i, k}^{(u)}\left(e^{j \omega}\right)$ would correspond to the frequency response of the updated model between input $k$ and output $i$. A measure of similarity, $D_{k}^{i}$, between the two models relating input $k$ and output $i$ can be expressed as

$$
D_{i, k}=\frac{1}{\omega_{N}} \int_{0}^{\omega_{N}} \frac{\min \left(\left|\mathbf{H}_{i, k}^{(f)}\left(e^{j \omega}\right)\right| \cdot\left|\mathbf{H}_{i, k}^{(u)}\left(e^{j \omega}\right)\right|\right)-c}{\max \left(\left|\mathbf{H}_{i, k}^{(f)}\left(e^{j \omega}\right)\right|,\left|\mathbf{H}_{i, k}^{(u)}\left(e^{j \omega}\right)\right|\right)-c} \mathrm{~d} \omega
$$

where $c$ is the smallest value evaluated in the interval $\left[0 . . \omega_{N}\right]$ for either function. This metric also ranges from 0 to 1 , with 1 suggesting very similar dynamic interactions between the designated input and output for the two models, and 0 suggesting otherwise. Consequently, since inputs into the model (5) are features extracted from sEMG signatures of the relevant muscles, overlaps from Eq. (6) depict how influences from various muscles on the corresponding dynamics change over time, as illustrated in Figure 5.

\section{Results}

The newly proposed methodology for monitoring of the NMS system was applied to track fatigue induced changes in the behavior of the NMS system of 12 human subjects. 
Muscle fatigue was used as a proof of concept because it is extensively studied and can be easily induced without damaging subjects. Conceptually, it can be seen as the inability for muscles to provide the required or expected force and, as such, corresponds to a degraded functioning of the NMS system. Therefore, tracking and characterizing fatigue via monitoring the NMS system seemed to be a plausible way to demonstrate the newly introduced methods.

While the precise definitions of fatigue are still debated (Westerblad, Allen and Lännergren, 2002), many reproducible NMS phenomena are associated with fatigue. Within the context of the proposed NMS modeling strategy, fatigue has been found to:

- Decrease sEMG frequencies due to active motor units firing more slowly and synchronously (Allison and Fujiwara, 2002);
- Occasionally increase sEMG amplitudes because of synchronous firing of active motor units (Allison and Fujiwara, 2002);

- Change muscle coordination (Corcos, Jiang, Wilding and Gottlieb, 2002);

- Change repetitive motion trajectories (Côté, Mathieu, Levin, and Feldman, 2002);

- Occasionally change local joint stability, which could arise from decreased proprioception (Myers, Guskiewicz, Schneider and Prentice, 1999), decreased kinesthesia (Pederson, Helstrom, Djupsjobacka and Johansson, 1999), increased reaction time (Edward, Bradford and Huston, 1996), and increased central nervous system processing time (van Duinen, Renken, Maurits and Zijdewind, 2007);

Each of the aforementioned physiological effects of fatigue will have an effect on the parameters in (5), leading to a natural validation of the techniques introduced in this paper.

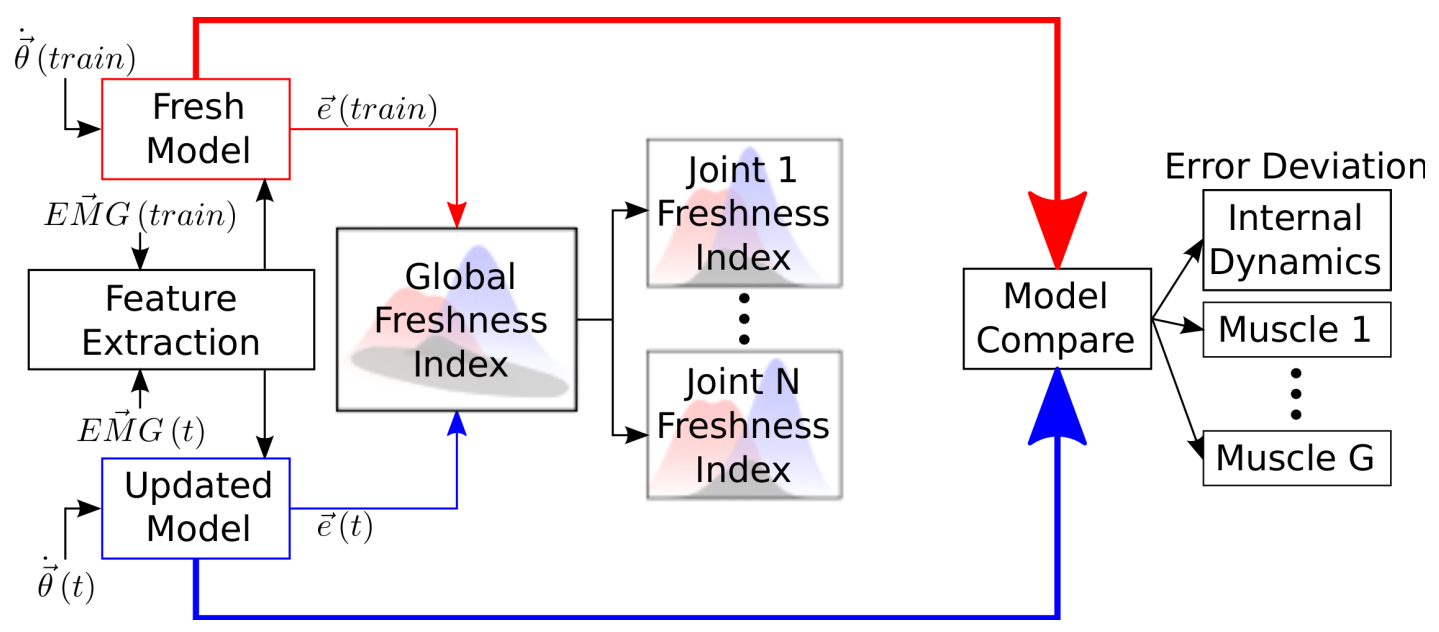

Figure 5. Flow chart of using model-based characterization of NMS degradation at the muscle level.

\subsection{Data Description}

The data used to demonstrate the new methodology was collected from 12 subjects in a study reported by Gates and Dingwell (2008). For the sake of completeness, the data collection procedure will be briefly described below, though more details can be found in (Gates and Dingwell, 2008). As illustrated in Figure 6, subjects were strapped into a high back chair with a five-point harness to reduce trunk motion. The subjects then performed a repetitive sawing motion at approximately $1 \mathrm{~Hz}$, as directed by a metronome, moving their hand in an anterior-posterior direction, while gripping a handle attached to a weighted sled. The sled was weighted to $15 \%$ of the subject's average maximum voluntary contraction force and each subject exercised until his/her voluntary exhaustion. During those exercises, relevant muscle EMGs and joint kinematic variables were sampled synchronously, as illustrated in Figure 7.
Before presenting the results, let us mention that in the proceeding sections, the statistical significance of trends in the EMG features, overlap coefficients GFI and JFI, and the muscle-joint overlap coefficients $D$ introduced by (6), was assessed via a $T$ test of the linear slope fits, at the $95 \%$ confidence level (Montgomery, Runger and Hubele, 2011).

\subsection{Feature Extraction}

Figure 8 depicts the sEMG features, $\left\langle f^{0} \mid t\right\rangle$ and $\left\langle f^{1} \mid t\right\rangle$ for one of the subjects in the study. Independent analysis of these features over time was conducted since literature indicates that they could be seen as indicators of fatigue in the sEMG signals (Allison and Fujiwara, 2002). Namely, decreasing instantaneous frequencies, $\left\langle f^{1} \mid t\right\rangle$, are considered to be a classic fatigue indicator in sEMG signals. We noted statistically negative linear trends in instantaneous frequencies in at least one muscle EMG for each of the subjects considered in this study. More specifically, 
statistically significant negative trends in the instantaneous frequency of the sEMG signals over time were present in $75 \%$ of the subjects for MT, PD, LD, AD, T and FCR, while sEMGs from B, PM, and ECRL muscles showed this trend in $58 \%$ of the subjects.

Increasing instantaneous intensities $\left\langle f^{0} \mid t\right\rangle$ in sEMG signals happen to be a less widely accepted fatigue indicator (Disselhorst-Klug, Schmitz-Rode and Rau, 2009). Nonetheless, we still noted that statistically significant positive linear trends in $\left\langle f^{0} \mid t\right\rangle$ over time occurred in at least one muscle for each subject. Specifically, statistically significant positive trends in the instantaneous energy of the sEMG signals were present in $67 \%$ of subjects for B, $50 \%$ of subjects for FCR, $42 \%$ of subjects for T and ECRL, $25 \%$ of the subjects for MT, PD, PM, and $\mathrm{AD}$, and $17 \%$ of subjects for LD.

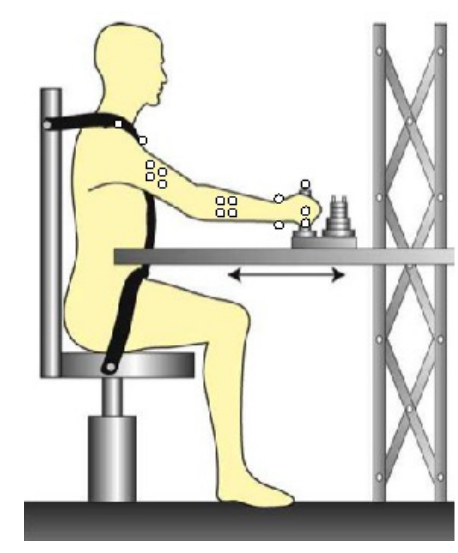

Figure 6. Seated subject executing sawing motion. White circles represent kinematic markers used to track the subject's motion via motion analysis system.

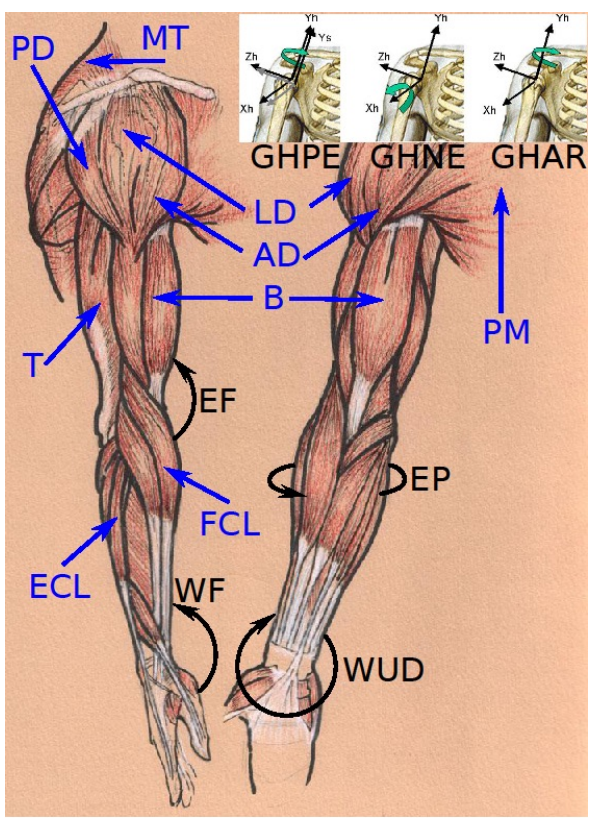

\begin{tabular}{|l|l|}
\hline Angle & Abbreviation \\
\hline Glenohumeral plane of elevation & GHPE \\
\hline Glenohumeral negative elevation & GHNE \\
\hline Glenohumeral axial rotation & GHAR \\
\hline Elbow flexion & EF \\
\hline Elbow pronation & EP \\
\hline Wrist ulnar deviation & WUD \\
\hline
\end{tabular}

\begin{tabular}{|l|l|}
\hline Muscle & Abbreviation \\
\hline Middle trapezius & MT \\
\hline Posterior deltoid & PD \\
\hline Lateral deltoid & LD \\
\hline Anterior deltoid & AD \\
\hline Pectoralis major & PM \\
\hline Biceps & B \\
\hline Triceps & T \\
\hline Flexor carpi radialis & FCR \\
\hline Extensor carpi radialis & ECR \\
\hline
\end{tabular}

Figure 7. Joint kinematic variables (black) and muscles (blue) for which measurements were acquired, superimposed on the anatomy of the human arm (adapted from Wu, van der Helm, Veeger, Maksous, Van Roy, Anglin, Nagels, Karduna, McQuade, Wang, Werner and Bucholtz, 2005). Joint kinematic data was collected at $60 \mathrm{~Hz}$ using an 8 camera, Vicon-612 motion analysis system from Oxford Metrics in Oxford, UK. Synchronously, sEMG data was captured at 1,080 Hz using Delsys system from Delsys, Inc. in Boston, MA.

One should note that the discussion above is purely based on the analysis of the inputs into the system and is thus symptomatic rather than system based in its nature. Let us now elaborate on what was found as we built and analyzed dynamic models relating the aforementioned sEMG features to the relevant NMS kinematic variables.

\subsection{Model Based Characterization of NMS Performance Degradation}

Figure 9 depicts the GFIs for the same subject considered in Figure 8. It clearly shows negative trend of this subject's GFIs over time. Actually, statistically significant decreasing trends in the GFIs over time were established for all 12 subjects. The median drop in GFIs over all subjects was $39 \%$, ranging between $15 \%$ and $64 \%$. 
Figure 10 shows the behavior of joint level overlap coefficients JFI over time for the same subject considered in Figures 8-9, with red lines indicating situations when statistically significant negative trends were noted. Overall, it was observed that negative trends in JFIs for GHPE, GHAR, EP, and WUD were statistically significant for all subjects. In addition, negative JFI trends for GHNE and WF were significant for all but one subject, while negative trends for the JFIs of EF were significant for all but three subjects. These prevalent downward trends are commensurate with the slow fatigue dynamics noted by Dingwell et al., (2007) and Segala et al. (2011).

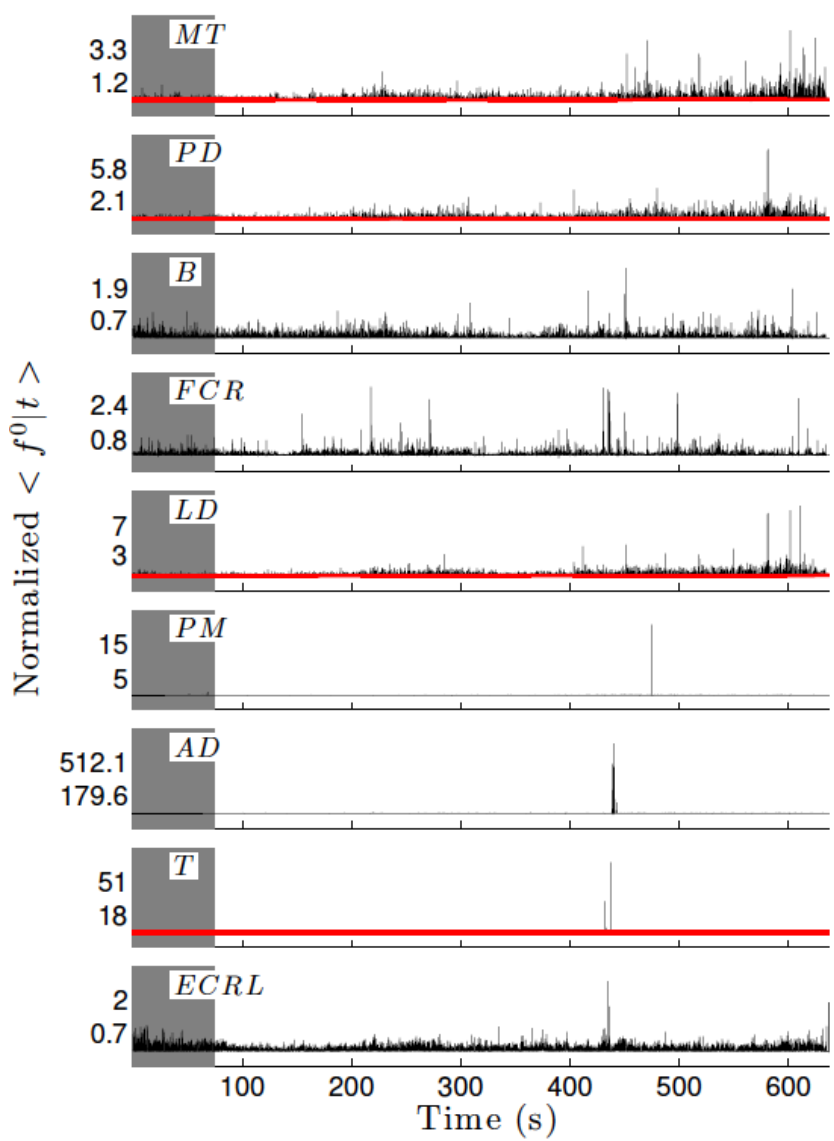

Besides trends in GFIs and JFIs, highly localized drops, like those occurring in Figures 9-10 at 135s, 246s, 314s, and especially $440 \mathrm{~s}$, were present at some point in time in each subject's GFIs and JFIs. They arise for two reasons. First, it is known that motion capture systems are imperfect and there are moments when it lost track of a marker. Many of these artifacts were filtered via cubic spline interpolant, minimizing the distances between markers in a cluster and a discrete wavelet filtering technique (Huzurbazar, Statistics and Doundation, 2013). However, it was obvious to the authors that the algorithms did not capture all artifact instances for all subjects. E.g., a typical example of this type of an event caused the drop at 246s in Figures 9 and 10.
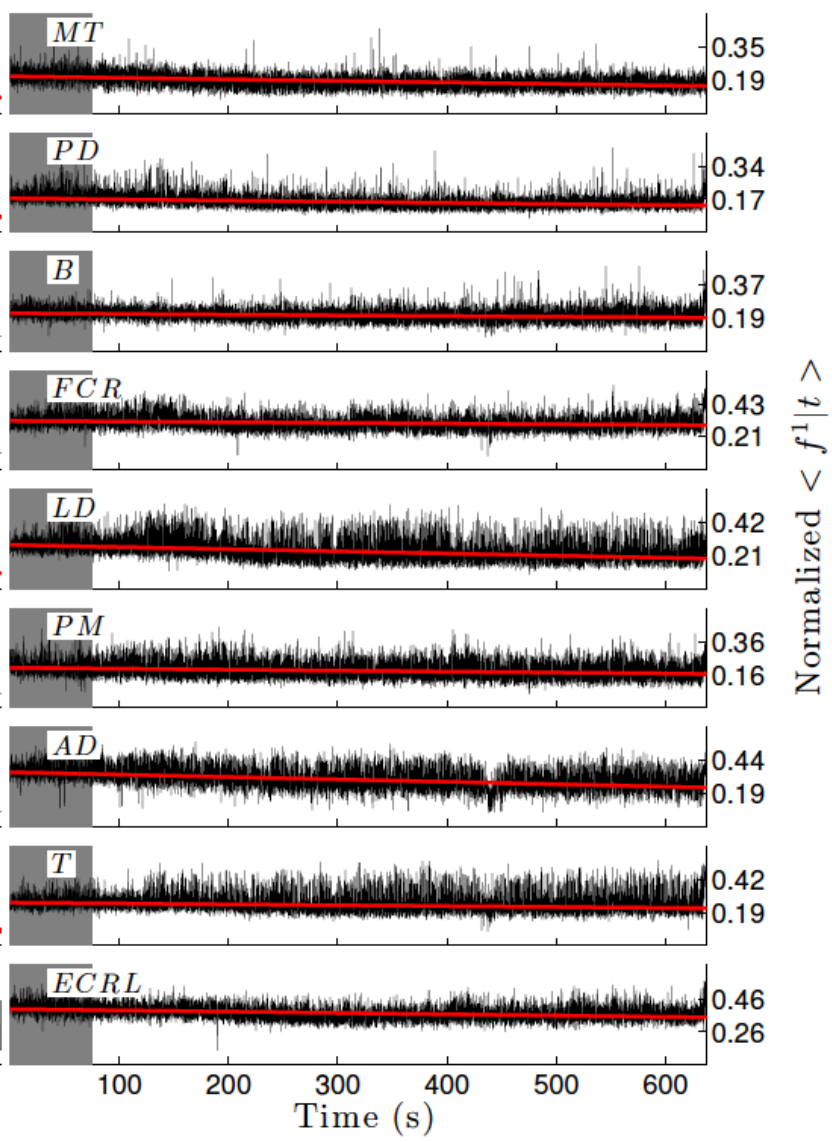

Figure 8. The left column of plots in this figure shows $\left\langle\boldsymbol{f}^{\mathbf{0}} \mid \boldsymbol{t}\right\rangle$ features, while The right column of plots shows $\left\langle\boldsymbol{f}^{\mathbf{1}} \mid \boldsymbol{t}\right\rangle$ features. In both columns, significant negative trends at the $95 \%$ level indicated with a bold red line. Please note that gray patches on the left side of each plot denote the fresh data. For each subject, the values of $\left\langle\boldsymbol{f}^{\mathbf{0}} \mid \boldsymbol{t}\right\rangle$ of any given muscle were scaled to range from 0 to 1 within the fresh data and these scaling factors were then applied to the rest of the data from that muscle. As for the values of $\left\langle\boldsymbol{f}^{\mathbf{1}} \mid \boldsymbol{t}\right\rangle$, they were scaled by the Nyquist frequency over the entire dataset. 


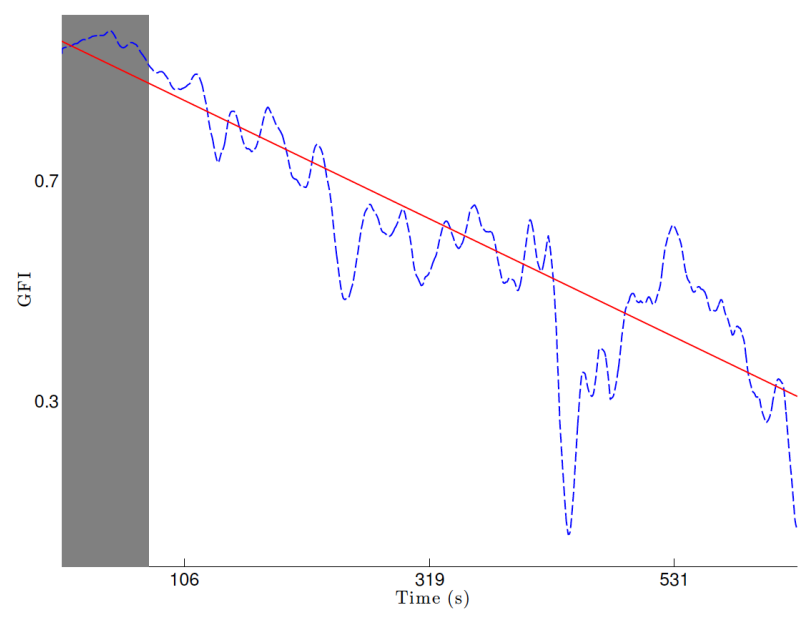

Figure 9. GFI over time for a selected subject. The red line represents a statistically significant negative trend at the $95 \%$ confidence interval.

Secondly, every subject, at least at some point in time displayed unusual muscular firing patterns during which for a period of time, one or more muscles attained instantaneous energies of up to two orders of magnitude greater than those seen in the fresh data. Since the model is trained only on the EMG signatures in the fresh data, such unusual input patterns occasionally led to the model briefly losing accuracy, which caused the overlap indices to temporarily drop. In fact, $1 / 3$ of subjects experienced instantaneous energies from 3 to 5 times those observed in the fresh data, another third of subjects experienced instantaneous energies an order of magnitude greater than what was seen in the fresh data and the remainder experienced two orders of magnitude greater instantaneous energies than those seen in the fresh data. A typical example of this type of an event is responsible for the drops at 440s in Figures 9 and 10, during which unusual patterns of muscular activity in the MT, B, FCR, LD, AD, T, and ECRL where observed, as visible in Figure 8. Nevertheless, despite these localized drops and recoveries, the general downward trend of overlap indices persists for all subjects.

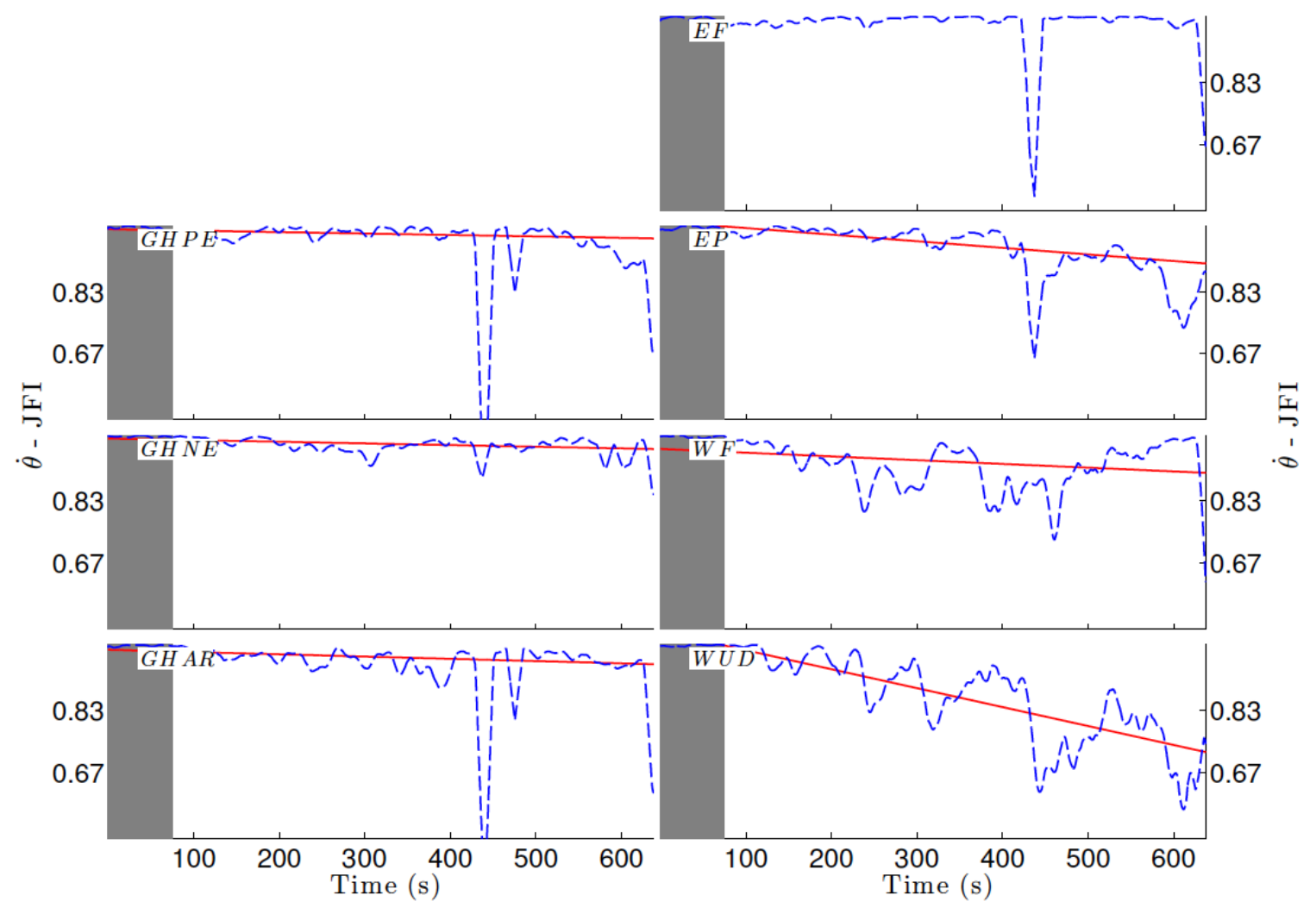

Figure 10. Individual JFI versus time for an example subject. The red lines represent statistically significant negative trends. 


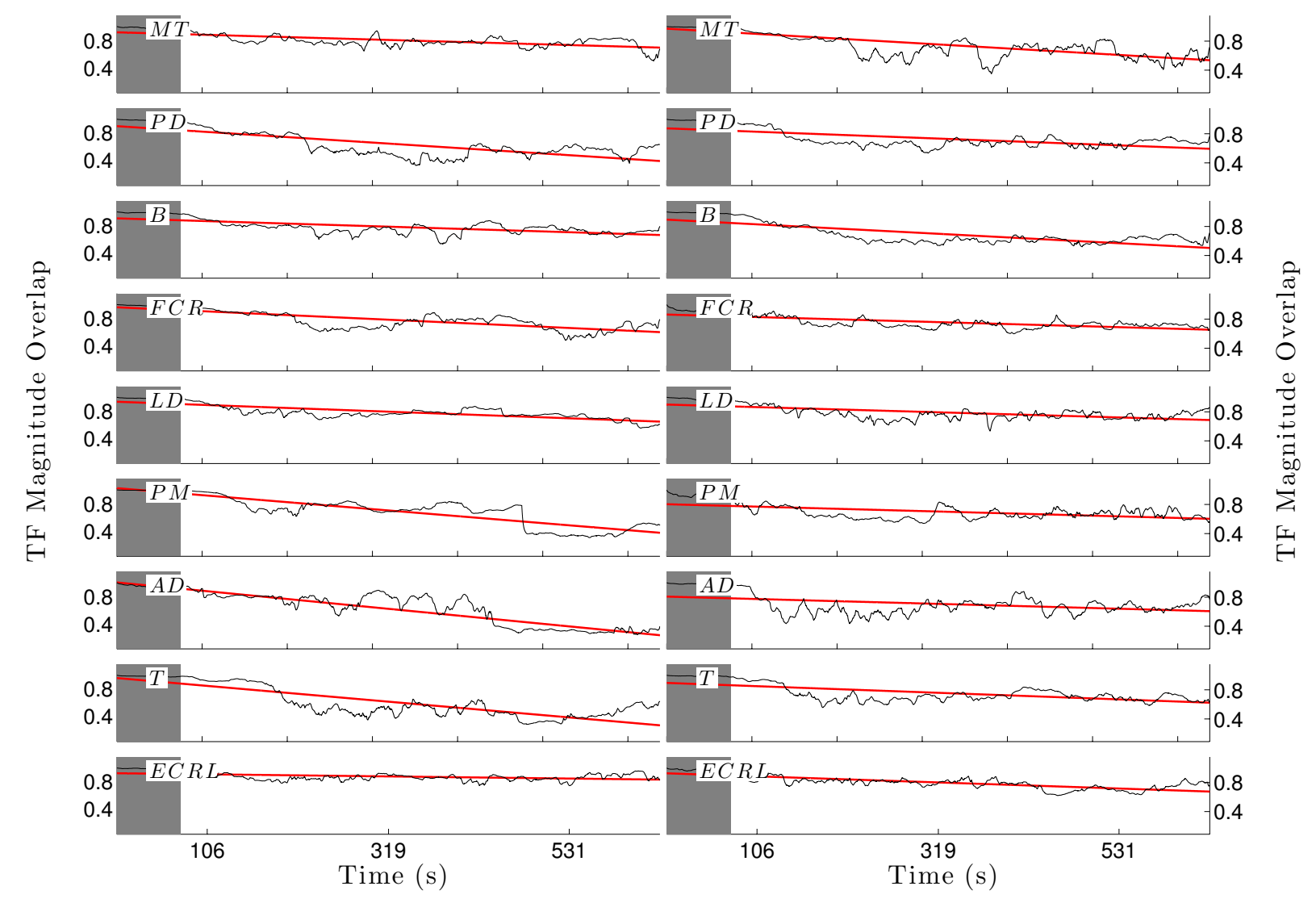

Figure 11. Overlap of transfer functions between EMG inputs to GHAR joint velocity for the fresh model and updated model. Gray patches represent fresh data. Transfer function overlaps for the $\left\langle\boldsymbol{f}^{\mathbf{0}} \mid \boldsymbol{t}\right\rangle$ inputs are in the left column of plots, while transfer function overlaps for the $\left\langle\boldsymbol{f}^{\mathbf{1}} \mid \boldsymbol{t}\right\rangle$ inputs are in the right column of plots.

Now, let us discuss the results of characterizing the degradation of the NMS system, using transfer function overlap coefficients $\mathrm{D}$ introduced by Equation (6). The reader should be reminded that these coefficients evaluate how influences of signatures from each individual muscle on each individual joint velocity changed over time. For the same subject that was considered in Figures 8-10, Figure 11 shows the coefficients D for the EMG inputs to the GHAR angular velocity, with red lines indicating statistically significant negative linear trends in those coefficients over time. It is obvious that all indices in Figure 11 show significant decreasing trends, illustrating a continuous change in the influences of the corresponding muscle features to GHAR velocity. Over all subjects, sEMG features, and joints, $96 \%$ of the transfer function overlap coefficients D exhibited statistically negative trends. Moreover, the muscles MT, $\mathrm{AD}$, and $\mathrm{LD}$ had negative trends for all joint velocities, over all subjects.

An especially interesting observation was that the length of time to the moment when subjects gave up further exercise seems to correlated with the behavior of the transfer function overlap coefficients D. Specifically, the 7 subjects who were able to exercise for the shortest duration of time showed negative linear trends in coefficients D for all muscles and all joints. All of the muscle signature/joint pairs that did not show negative trends were restricted to the 5 subjects who exercised the longest. Furthermore, the two longest performing subjects did the exercise two times longer than the next longest performing subject, and exhibited more than $82 \%$ of the muscle signature/joint combinations that did not show decreasing trends in the transfer function overlaps (one of those two was a triathlete). These results seem to indicate that our systembased approach is confirming that the two subjects who exercised the longest had more endurance and fatigued to a lesser degree.

\section{Conclusions AND Future Work}

This paper presents a system-based methodology for monitoring of NMS system performance. It uses a set of signatures extracted from the TFDs of sEMG signals and builds a dynamic model that relates these signatures to joint velocities. NMS system monitoring was then realized via statistical analysis of modeling errors and model parameters, as they change over time. 
The new monitoring approach was used to track and characterize fatigue-induced changes in the NMS system via an experiment involving 12 subjects performing a repetitive sawing motion until voluntary exhaustion. Error analysis of these models led to detection of statistically significant trends in the NMS system models for all subjects. Furthermore, the proposed system detected statistically significant trends in the distributions of modeling errors yielded by models of $96.5 \%$ of the 84 angular joint velocities considered in this study ( 7 joint velocities for each of the 12 subjects). In addition, a statistically significant monotonous change in the effects of muscle activity on the joint motion was evident in $100 \%$ of subjects, with statistically significant linear decreasing trends detected in $96 \%$ of the overlaps of transfer functions between EMG signatures and joint velocities, over all subjects. Finally, it was observed that the subjects who performed the exercise the longest showed the least degradation in terms of changes in the dynamic relationships between the muscle signatures and joint velocities.

The ability of the newly proposed monitoring methodology to track and characterize changes in the NMS system can eventually become useful in personalizing and customizing training regimens. E.g., for athletes, muscles that fatigue most quickly can be identified and training regimens can be built to address these deficits. Similarly, therapeutic exercise regimens for patients with NMS impairments or patients undergoing rehabilitation can be tailored toward returning the patient to a nominally healthy set of joint dynamics. Furthermore, the detection and characterization of fatigue can be used as an indication to stop training or rehabilitation before the onset of injury.

Several avenues for future research are apparent. First, and most importantly, the linear dynamics assumption should be lifted and a nonlinear NMS model appropriate for its monitoring should be pursued. Furthermore, there are possibilities for better utilization of the information contained in the EMG TFDs, such as the use of higher order instantaneous frequency statistics or entropies of the TFDs. In addition, fatigue is known to increase muscle EMD (Zhou, Carey and Snow, 1998) and it would be advantageous to design a method to track these changes within the dynamic NMS models developed in this work. Finally, the capabilities of the newly introduced technique should be further explored on data corresponding to less constrained motions, such as walking or running.

\section{ACKNOWLEDGEMENT}

Research reported in this publication was supported in part by National Institute of Biomedical Imaging and Bioengineering of the National Institutes of Health (NIH), under award number EB003425. This research is also supported in part by the National Science Foundation (NSF) grant IIP 1266279. The content is solely the responsibility of the authors and does not necessarily represent the official views of the NIH or NSF.

\section{REFERENCES}

Allison, G. T. \& Fujiwara, T. (2002). The relationship between EMG median frequency and low frequency band amplitude changes at different levels of muscle capacity. Clinical Biomechanics (Bristol, Avon). 17(6), pages 464-469.

Arslan, Y. Z., Adli, M. A., Akan A. \& Baslo, M. B. (2010). Prediction of externally applied forces to human hands using frequency content of surface EMG signals. Computer Methods and Programs in Biomedicine, 98(1), pages 36-44.

Artemiadis P. K. \& Kyriakopoulos, K. J. (2009). EMGbased position and force estimates in coupled humanrobot systems: towards EMG-controlled exoskeletons. In Khatib, O., Kumar, V \& Pappas, G. (Eds.), Experimental Robotics - The Elevents International Symposium (pages. 241-250). Berlin-Heidelberg: Springer-Verlag.

Asfour, T., Berns, K., Schelling, J. \& Dillmann, R. (1999). Programming of manipulation tasks of the humanoid robot ARMAR. Proc. of the 9th International Conference on Advanced Robotics (pages 107-112), Oct. 25-27, 1999, Tokyo, Japan.

Atkinson K. A. (1989). An Introduction to Numerical Analysis. New York, NY, John Wiley \& Sons $\left(2^{\text {nd }}\right.$ Edition).

Bäck, T. (1996). Evolutionary Algorithms in Theory and Practice. New York City, NY: Oxford University Press.

Buchanan, T.S., Lloyd, D. G., Manal, K. \& Besier, T.F. (2005). Estimation of Muscle Forces and Joint Moments Using a Forward-Inverse Dynamics Model. Medicine \& Science in Sports \& Exercise. 37(11), pages 1911-1916.

Cohen, L. (1995). Time Frequency Analysis. Englewood Cliffs: Prentice-Hall, Inc.

Corcos, D. M., Gottlieb, G. L., Latash, M. L., Almeida, G. L. \& Agarwal, G. C. (1992). Electromechanical delay: an experimental artifact," Journal of Electromyography and Kinesiology. 2(2), pages 59-68.

Corcos, D. M., Jiang, H.-Y., Wilding, J. \& Gottlieb, G. L. (2002). Fatigue induced changes in phasic muscle activation patterns for fast elbow flexion movements. Experimental Brain Research, 142(1), pages 1-12.

Côté, J. N., Mathieu, P. A., Levin, M. F. \& Feldman, A. G. (2002). Movement reorganization to compensate for fatigue during sawing. Experimental Brain Research. 146(3), pages 394-398.

De Jong, K. A. (1975). Analysis of the behavior of a class of genetic adaptive systems. Doctoral Dissertation, 
University of Michigan, Ann Arbor, MI, URL: http://deepblue.lib.umich.edu/handle/2027.42/4507

Deep, K., Singh, K. P., Kansal, M. \& Mohan, C. (2009). A real coded genetic algorithm for solving integer and mixed integer optimization problems. Applied Mathematics and Computation. 212(2), pages 505-518.

Dingwell, J. B., Napolitano, D. F. \& Chelidze, D. (2007). A nonlinear approach to tracking slow-time-scale changes in movement kinematics. Journal of Biomechanics. 40(7), pages 1629-1634.

Disselhorst-Klug, C., Schmitz-Rode, T. \& Rau, G. (2009). Surface electromyography and muscle force: limits in sEMG-force relationship and new approaches for applications. Clinical Biomechanics. 24(3), pages 225235.

Edward, M., Bradford, B. \& Huston, L. J. (1996). The effects of muscle fatigue on neuromuscular function and anterior tibial translation in healthy knees. The American Journal of Sports Medicine. 24(5), pages 615-621.

Franklin, G., Powell J. \& Emami-Naeini, A. (2006). Feedback Control of Dynamic Systems. Upper Saddle River, NJ: Pearson Prentice Hall ( $5^{\text {th }}$ Edition).

Gates, D. H. \& Dingwell, J. B. (2008). The effects of neuromuscular fatigue on task performance during repetitive goal-directed movements. Experimental Brain Research, 187(4), pages 573-585.

Hashemi, J., Hashtrudi-Zaad, K., Morin, E. \& Mousavi, P. (2010). Dynamic modeling of EMG-force relationship using parallel cascade identification. Proc. of the IEEE International Conference of the Engineering in Medicine and Biology Society (pages pp. 1328-1331), Aug. 31-Sep. 4, 2010. Buenos Aires, Argentina.

Hurvich, C. M. \& Tsai, C.-L. (1991). Bias of the corrected AIC criterion for underfitted regression and time series models, Biometrika, 78(3), pages 499-509.

Huzurbazar, S., Statistics, G. \& Foundation, I. (2013). Wavelet-based detection of outliers in time series. Journal of Computational and Graphical Statistics. 11(2). pages 311-327.

Itoh, Y., Akataki, K., Mita, K., Watakabe, M. \& Nonaka, H. (2013). Frequency response model of skeletal muscle and its association with contractile properties of skeletal muscle. Journal of Electromyography and Kinesiology. 23(3), pages 572-579.

Jeong J. \& Williams, W. J. (1992). Kernel design for reduced interference distributions. IEEE Transactions on Signal Processing. 40(2), pages 402-412.

Keynes, R. D., Aidley, D. J. \& Huang, C. L. (2010). Nerve and Muscle. 4th Edition. New York, NY: Cambridge University Press.
Koike, Y. \& Kawato, M. (1995) Estimation of dynamic joint torques and trajectory formation from surface electromyography signals using a neural network model. Biological Cybernetics, 73(4), pages 291-300.

Lee, D., Glueck, M., Khan, A., Fiume, E., \& Jackson, K. (2010). A survey of modeling and simulation of skeletal muscle. ACM Transactions on Graphics. 28(4), doi. 10.1145/1559755.1559763

Lindsay, B. G. (1995). Mixture models: theory, geometry and Applications (pages 1-6). Hayward, CA: Institute of Mathematical Statistics.

Manal, K. (2003). A one-parameter neural activation to muscle activation model: estimating isometric joint moments from electromyograms, Journal of Biomechanics. 36(8), pages 1197-1202.

Matusita, K. (1955). Decision rules, based on the distance, for problems of fit, two samples, and estimation. The Annals of Mathematical Statistics. 26 pages 631-640.

Montgomery, D. C., Runger G. C. \& Hubele, N. F. (2011) Engineering Statistics, John Wiley \& Sons ( $5^{\text {th }}$ Edition).

Myers, J. B., Guskiewicz, K. M., Schneider R. A. \& Prentice W. E. (1999). Proprioception and neuromuscular control of the shoulder after muscle fatigue. Journal of Atheletic Training 34(4), pages 362367.

Pederson, J., Lonn, J., Helstrom, F., Djupsjobacka, M. \& Johansson, H. (1999). Localized muscle fatigue decreases the acuity of the movement sense in the human shoulder. Medicine \& Science in Sports \& Exercise. 31(7), pages 1047-1052.

Potvin J. R. \& Brown, S. H. M. (2004). Less is more: high pass filtering, to remove up to $99 \%$ of the surface EMG signal power, improves EMG-based biceps brachii muscle force estimates. Journal of Electromyography and Kinesiology. 14(3), pages 389-399.

Segala, D. B., Gates, D. H. Dingwell, J. B. \& Chelidze, D. (2011). Nonlinear smooth orthogonal decomposition of kinematic features of sawing reconstructs muscle fatigue evolution as indicated by electromyography. Journal of Biomechanical Engineering. 133(3), doi: 10.1115/1.4003320

Song, R. \& Tong, K. Y. (2005). Using recurrent artificial neural network model to estimate voluntary elbow torque in dynamic situations. Medical \& Biological Engineering \& Computing. 43(4), pages 473-480.

van Duinen, H., Renken, R., Maurits, N. \& Zijdewind, I. (2007). Effects of motor fatigue on human brain activity, an fMRI study. NeuroImage. 35(4), pages 1438-1449.

Viceconti, M., Testi, D., Taddei, F., Martelli, S., Clapworthy G., \& Jan, S. (2006). Biomechanics modeling of the musculoskeletal apparatus: status and 
key issues. Proceedings of the IEEE. 94(4), pages 725739.

Westerblad, H. K., Allen D. \& Lännergren J. (2002). Muscle fatigue: lactic acid or inorganic phosphate the major cause? Physiology. 17(1), pages 17-21.

Wu, G., van der Helm, F. C. T., Veeger, H. E. J., Makhsous, M., Van Roy, P., Anglin, C., Nagels, J., Karduna, A. R., McQuade, K., Wang, X., Werner, F. W. \& Buchholz, B. (2005). ISB recommendation on definitions of joint coordinate systems of various joints for the reporting of human joint motion - Part II: shoulder, elbow, wrist and hand. Journal of Biomechanics. 38(5), pages 981-992.
Xu, J.X., Zhang, Y. \& Pang, Y.J. (2010). A nonlinear parametric identification method for biceps muscle model by using iterative learning approach. Proc. of the IEEE International Conference on Control and Automation (pages 252-257), June 9-11, 2010. Xiamen, China.

Zhou, S., Carey, M. \& Snow, R., (1998). Effects of muscle fatigue and temperature on electromechanical delay. Electromyography and Clinical Neurophysiology. 38(2),pages 67-73. 